\title{
Hydrogel films of membrane type for biomedical application on the basis of polyvinylpyrrolidone copolymers
}

\author{
Yuriy Melnyk $^{1}$, Galyna Yatsulchak ${ }^{1}$, Sofiia Suberliak ${ }^{2}$, Romana Petrina $^{2}$, Volodymyr Skorokhoda $^{1}$ \\ Lviv Polytechnic National University; 12, S. Banbera Str., Lviv, 79013, Ukraine \\ ${ }^{1}$ Department of Chemical Technology of Plastics Processing; E-mail: yuriy.ya.melnyk@lpnu.ua \\ ${ }^{2}$ Department of Technology of Biologically Active Compounds, Pharmacy and Biotechnology
}

\begin{abstract}
The kinetic of copolymerization of 2-hydroxyethyl methacrylate (HEMA) with polyvinylpyrrolidone (PVP) in aqueous-organic media was investigated. The optimal initiating systems and the temperature regimes of the polymerization for fabrication of hydrogel film membranes based on HEMA/PVP copolymers were developed. The influence of the structure of the hydrogel film membranes synthesized with rare cross-linked copolymers on their the basic operational properties was established.
\end{abstract}

Keywords - polyvinylpyrrolidone, cross-linked copolymer, hydrogel, film membrane.

\section{Introduction}

Hydrogel materials based on 2-hydroxyethyl methacrylate with polyvinylpyrrolidone rarely cross-linked copolymers are used effectively for the manufacturing of contact lenses, polymer carriers of drugs, medical bandages, removable dentures in dentistry, as implants in plastic surgery etc [1] - [3]. Such materials are usually used in the form of films, which, at the same time, serve as membrane functions. The most common method for obtaining film membrane hydrogels is the polymerization of compositions in an aqueous medium or a watersoluble organic solvent, which after synthesis is replaced with water or aqueous solution [3], [4] . Operational and technological properties of hydrogel copolymers, including permeability and sorption ability, are largely determined by their composition and structural parameters of the network. Therefore, it is important to search for effective methods of directed formation of the structure of copolymers, which will allow to predict the operational properties of hydrogels on their basis. The aim of the work was to develop the foundations of technology and modes of formation of hydrogel membranes based on compositions of HEMA with PVP with an adjustable structure and composition of copolymers.

\section{Experimental Set-up and Procedure}

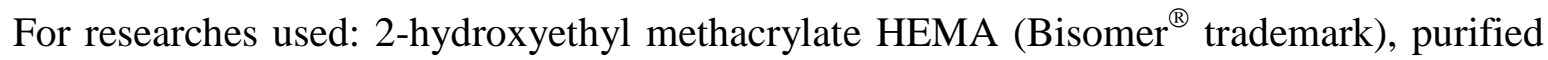
by distillation in a vacuum (the boiling points is $78^{\circ} \mathrm{C}$, under a residual pressure is $130 \mathrm{~N} / \mathrm{m}^{2}$ ); polyvinylpyrrolidone PVP10 with a molecular weight of $10 \cdot 10^{3}$ (SIAL Sigma-Aldrich ${ }^{\circledR}$ trademark) pharmaceutical purification. Experimental samples of hydrogel film membranes were obtained by copolymerization HEMA with PVP in a solvent medium between two glass plates. The distance between the plates determined the required thickness of the film. The kinetics of polymerization was studied by the chemical method by reducing the amount of unreacted monomer. The fraction of PVP, which entered into the reaction of grafted polymerization, was determined by photocolorimetric method. The sorption properties of hydrogels with respect to water were determined by the weight method, the mechanical properties - by the method of film breakage in the aqueous medium, the permeability of the membranes for water and dissolved substances in it - by the method of osmosis. 


\section{Results and discussions}

Studies in the kinetics of polymerization have found that the addition of PVP to the composition significantly increases the rate of polymerization. The polymerization is affected by the physical interaction between the components of the reaction medium through the so-called "matrix effect" with the formation of a charge-transfer complex. To investigate the initiator's influence on the polymerization of HEMA in the presence of PVP and the choice of the optimal initiator and its amount, the reaction was initiated by benzoyl peroxide (BPO), potassium persulfate (KPS) and azo-bis-isobutyric acid dinitrile (AAD). The rate of polymerization increases in the series AAD - BPO-KPS. For peroxide initiators, the rate of polymerization reaction is greater, which is obviously due to the promotional effect of PVP on the decomposition of peroxides.

Investigations of the influence of the nature of the solvent on the polymerization process revealed that the highest polymerization rate of HEMA in the presence of PVP is observed in an aqueous medium. In water, the compositions polymerize at high speed, even with a large dilution with a solvent at low temperatures $\left(55 \ldots 65^{\circ} \mathrm{C}\right)$. This enables the synthesis of hydrogel membranes on their basis in mild conditions and to avoid unwanted exothermic effects. On the basis of kinetic studies, the two-stage mode of formation of film hydrogels is substantiated: 1 stage $-55^{\circ} \mathrm{C}(2,5$ hours $), 2$ stages $-70{ }^{\circ} \mathrm{C}$ ( 3 hours).

Hydrogels on the basis of copolymers PVP with HEMA are cross-linked and consist of macromolecules PVP, to which grafted chains polyHEMA. The course of graft polymerization is confirmed by IR spectroscopy, differential-thermal and thermogravimetric analyzes. For all compositions with different contents of PVP there is an increase in efficiency and an extreme change in the degree of grafting over time.

The porosity of film hydrogels, which determines their permeability, can be controlled by polymerization in the presence of different amounts of solvents (Table 1). In this case, the porosity depends on the amount of solvent in the initial composition for the constant ratio monomer: PVP.

Table 1

Influence of composition content, nature and amount of solvent on the structure and properties of hydrogel membranes (polymerization regime: $55{ }^{\circ} \mathrm{C}-2,5 \mathrm{~h}, 70{ }^{\circ} \mathrm{C}-3 \mathrm{~h}$; [KPS] $=0,25 \%$ mass)

\begin{tabular}{|c|c|c|c|c|c|c|c|c|}
\hline \multicolumn{4}{|c|}{ Content of the components, mass p. } & \multirow[b]{2}{*}{$\begin{array}{c}M_{n}, \\
\mathrm{~kg} / \mathrm{mole}\end{array}$} & \multirow[b]{2}{*}{$\begin{array}{c}\sigma, \\
\mathrm{MPa}\end{array}$} & \multirow[b]{2}{*}{$\begin{array}{l}W, \\
\%\end{array}$} & \multicolumn{2}{|c|}{ Permeability coefficient* } \\
\hline HEMA & PVP & $\mathrm{H}_{2} \mathrm{O}$ & DMSO & & & & $\begin{array}{l}k_{\mathrm{H}_{2} \mathrm{O}} \cdot 10^{3}, \\
\mathrm{~m}^{3} /\left(\mathrm{m}^{2} \cdot \mathrm{h}\right)\end{array}$ & $\begin{array}{c}k_{\mathrm{NaCl}}, \\
\text { mole } /\left(\mathrm{m}^{2} \cdot \mathrm{h}\right)\end{array}$ \\
\hline 10 & - & 10 & - & 12 & 0,53 & 42 & 0,51 & 80 \\
\hline 9 & 1 & 10 & - & 20 & 0,46 & 45 & 2,90 & 141 \\
\hline 8 & 2 & 10 & - & 24 & 0,40 & 48 & 5,23 & 181 \\
\hline 8 & 2 & 9,9 & 0,1 & 25 & 0,40 & 52 & 5,30 & - \\
\hline 8 & 2 & 9 & 1 & 34 & 0,41 & 47 & 5,65 & 212 \\
\hline 8 & 2 & 8 & 2 & 46 & 0,41 & 47 & 6,24 & 240 \\
\hline 8 & 2 & - & 10 & 63 & 0,42 & - & 7,10 & 272 \\
\hline 8 & 2 & 20 & - & 28 & 0,38 & 55 & 7,42 & 234 \\
\hline 7 & 3 & 10 & - & 38 & 0,31 & 53 & 7,14 & 232 \\
\hline 5 & 5 & 10 & - & 51 & 0,22 & 61 & 10,2 & 274 \\
\hline
\end{tabular}

DMSO - dimethyl sulfoxide; $M_{n}$-internodal molecular mass; $\sigma$ - tensile strength;

$W$ - water content; * - thickness of the hydrogel film is $200 \mu \mathrm{m}$ 
The grid density, which is determined by the molecular weight of the internodal fragment $M_{n}$, is a measure of permeability in the case of a defect-free structure. However, the content of the solvent in the initial composition, which exceeds its maximum content during the equilibrium absorption of the polymer matrix, has a natural phase separation, which manifests itself in the turbidity of the film.

The researches have established that by selecting the nature of the solvent (protonodonor or aprotic, or a mixture thereof), it is possible to adjust the density of the mesh of the hydrogel membrane [4]. Changing the density of the net affects the permeability of the membrane for low molecular weight substances (Table 1). Strength during stretching of membranes at the same time practically does not change.

The greatest influence on the structural parameters of the grid and permeability was observed when small amounts of dimethyl sulfoxide were added to the water. These parameters were subsequently virtually unchanged for more than $30 \%$ by weight of dimethyl sulfoxide of total solvent. At the same time, hydrogel membranes based on HEMA and PVP copolymers are characterized not only by increased sorption properties (compared with homopolymers HEMA), which were estimated by water content but also several times more permeability for water and aqueous solution of a model substance (sodium chloride) (Table 1).

\section{Conclusions}

According to the proposed method for forming the structure of the hydrogel, the porosity of the membrane is directed to change the density of the polymer matrix grid, which results in a controlled change in permeability. The strength of the membranes based on HEMA-PVP copolymers at the same time vary slightly. The performed researches are the basis of the developed method of regulation of the structure and properties of hydrogels [4] and membranes on their basis.

\section{References}

[1] O. V. Suberlyak, V. Y. Skorokhoda, N. B. Semenyuk and Yu. Ya. Melnyk, Materialy biomedychnoho pryznachennia na osnovi (ko)polimeriv polivinilpirolidonu. Lviv: Publishing House of Lviv Polytechnic Nataional University, 2015.

[2] O. Y. Komarytsia, S. Yu. Kramarenko, N. M. Baran and Yu. Ya. Melnyk, "Vyvchennia khimichnoi kinetyky desorbtsii khlorheksydynu iz hidroheliu na osnovi adhezyvno aktyvnoho polimeru u skladi znimnoho tymchasovoho plastynkovoho protezu", Novyny Stomatolohii, no. 2 (95), pp. 80-84, 2018.

[3] V. Skorokhoda, Yu. Melnyk, N. Semenyuk, N. Ortynska and O. Suberlyak, "Film hydrogels on the basis of polyvinylpyrrolidone copolymers with regulated sorption-desorption characteristics", Chemistry \& Chemical Technology, vol. 11, no. 2, pp. 171-174, 2017.

[4] O. V. Suberlyak, Yu. Ya. Melnyk, V. Y. Skorokhoda, "Regularities of preparation and properties of hydrogel membranes", Materials Science, vol. 50, no. 6, pp. 889-896, 2015. 